\title{
Real-time deformation monitoring with GPS and Kalman Filter
}

\author{
Cankut D. Ince and Muhammed Sahin \\ Istanbul Technical University, Faculty of Civil Engineering, Department of Geodesy and Photogrammetry, 80620 Maslak, Istanbul, Turkey
}

(Received December 25, 1999; Revised June 30, 2000; Accepted June 30, 2000)

\begin{abstract}
The main purpose of this research is to develop a real-time GPS monitoring system with the aid of a Kalman Filter for use in an active tectonic region near Istanbul, and its surrounding region. Currently, an ongoing project exists, funded by the World Bank, that monitors deformation in Istanbul and the Marmara Region. Istanbul is one of the largest cities in the world, and is under possible earthquake threat. In order to set up a powerful control system, a surveying and estimation method was designed and the necessary software, called RT-MODS2 (Real-Time Monitoring Of Dynamic Systems 2), was developed. The software reads real-time input data from GPS receivers and performs deformation analyses with the help of the Kalman Filter. Some studies of filtering and deformation analysis were performed in order to detect failures and outliers, and to increase the reliability of the deformation analysis.
\end{abstract}

\section{Introduction}

At present, instead of static deformation monitoring approaches, continuous dynamic deformation monitoring methods have been increasingly used to understand natural events such as earthquakes and landslides, and to monitor the stability of manmade structures such as buildings, bridges, nuclear plants, dams, etc. (Leick, 1995; Bennet et al., 1998; Bock and Bevis, 1999). Real-time GPS technology is an important development to aid continuous deformation monitoring, where the timely detection of any deformation is critical. The kinematic/dynamic parameters of deformation are computed in order to the predict failure events. Hence the use of the Kalman Filter for the estimation of the state vector of a deformation object is very convenient (Grewal and Andrews, 1993). The elements of the state vector in the Kalman Filter are the unknowns of the kinematic/dynamic system. These are typically the position of the object, and the variation of the position. This property of the Kalman Filter is important for studying the behaviour of deformations, and especially for the investigation of catastrophes.

The system described in this study is the product of a pilot project, to be used as an a-priori warning system that continuously monitors deformation activity in and around Istanbul, a city which is under a large earthquake threat in the near future. The earthquake risk varies from 3 months to 30 years according to seismologists and geologists (Barka, 1999). The developed software, RT-MODS2, does not perform raw GPS data processing in its present form, but reads real-time GPS results, performs Kalman filtering, detects outliers and carries out deformation analysis (Ince, 1999). The input is the position data obtained from RTK GPS phase solutions, output in the so-called NMEA 0183 (National Marine Electronics Association) format (Langley, 1995).

Copy right (C) The Society of Geomagnetism and Earth, Planetary and Space Science (SGEPSS); The Seismological Society of Japan; The Volcanological Society of Japan; The Geodetic Society of Japan; The Japanese Society for Planetary Sciences.

\section{Kalman Filter}

The Kalman Filter was designed to estimate the linear dynamic systems (Kalman, 1960; Kalman and Bucy, 1961). According to Grewal and Andrews (1993), the Kalman Filter is an estimator for what is called the linear-quadratic Gaussian, while Maybeck (1979) claims that the Kalman Filter is simply an optimal recursive data processing algorithm.

The mathematical form of the Kalman Filter consists of two independent models, the dynamic and measurement models given by the following formulae (Kalman, 1960; Teunissen and Salzmann, 1988; Hofmann-Wellenhof et al., 1997; Leick, 1995):

$$
\begin{aligned}
x_{k} & =\phi_{k, k-1} x_{k-1}+w_{k} \\
l_{k} & =A_{k} x_{k}+V_{k}
\end{aligned}
$$

where;

$x_{k}$ is the state vector at time $t_{k}$

$x_{k-1}$ is the state vector at time $t_{k-1}$

$\phi_{k, k-1}$ is the transition matrix from time $t_{k-1}$ to $t_{k}$

$w_{k}$ is the noise vector representing the dynamic model at

time $t_{k}$

$l_{k}$ is the observation vector at time $t_{k}$

$A_{k}$ is the design matrix for the measurement model

$V_{k}$, is the noise vector, which represents the measurement model at time $t_{k}$.

Under the statistical assumptions described in Tsai and Kurz (1983) and Cross (1990), the prediction and filtering equations of the Kalman Filter can be summarized as follows.

The prediction equations:

$$
\begin{aligned}
\hat{x}_{k}^{-} & =\phi_{k, k-1} \hat{x}_{k-1} \\
Q_{k}^{-} & =\phi_{k, k-1} Q_{k-1} \phi_{k, k-1}^{T}+Q_{w} \\
Q_{w} & =\sigma_{a}^{2} w_{k} w_{k}^{T}
\end{aligned}
$$


The filtering equations:

$$
\begin{aligned}
\hat{x}_{k} & =\hat{x}_{k}^{-}+K_{k}\left(l_{k}-A_{k} \hat{x}_{k}^{-}\right) \\
Q_{k} & =\left(I-K_{k} A_{k}\right) Q_{k}^{-T} \\
K_{k} & =Q_{k}^{-} A_{k}^{T}\left(A_{k} Q_{k}^{-} A_{k}^{T}+Q_{l}\right)^{-1}
\end{aligned}
$$

where;

$\hat{x}_{k}^{-}$is the predicted estimate of the state vector at time $t_{k}$, $\hat{x}_{k-1}$ is the filtered estimate of the state vector at time $t_{k-1}$, $Q_{k}^{-}$is the covariance matrix of the predicted state vector, $Q_{w}$ is the covariance matrix of the dynamic model noise, $\sigma_{a}^{2}$ is the variance of the non-deterministic variable in the dynamic model,

$Q_{k}$ is the covariance matrix of the filtered state vector.

$K_{k}$ is the gain matrix.

Before deformation analysis can proceed, probable outliers should be first detected. The most important criterion for the detection of outliers in Kalman Filters is the innovation sequence (Salzmann, 1990):

$$
v_{k}=l_{k}-A_{k} \hat{x}_{k}^{-} .
$$

Outliers can be detected by application of the local and global slippage tests (Teunissen and Salzmann, 1988). However, RT-MODS2 determines outliers only with the local slippage test because it is more convenient and simpler to implement for real-time applications than the global slippage test. Local slippage tests at time $t_{k}$, depend only on the predicted state and the observations at time $t_{k}$ (Teunissen, 1990; Jin, 1996).

\section{Continuous Deformation Analysis and an Ap- plication}

Deformation analysis is, in general, performed using the results of certain epochs. However, the results at the beginning of the Kalman Filter may not be convenient for determining the kinematics/dynamics of the object. So in this study, deformations are detected according to the results of the 20th estimation interval onwards. For example, the test value for the height is:

$$
\begin{aligned}
& T_{i}^{h}=\frac{d_{i}^{h 2}}{\sigma_{d_{i}}^{2}} \quad i>20 \\
& d_{i}^{h}=h_{i}-h_{20} \quad i>20 \\
& \sigma_{d_{i}}^{2}=\sigma_{h h}^{i}+\sigma_{h h}^{20} \quad i>20
\end{aligned}
$$

where,

$$
\begin{aligned}
& T_{i}^{h} \text { is test value for the height deformation at the } i \text { th } \\
& \text { estimation interval, } \\
& d_{i}^{h} \text { is the difference vector at the } i \text { th estimation interval } \\
& \text { according to the } 20 \text { th estimation interval, } \\
& \sigma_{h h}^{i} \text { is the variance of the height estimation at the } i \text { th } \\
& \quad \text { estimation interval, } \\
& \sigma_{h h}^{20} \text { is the variance of the height estimation at the 20th } \\
& \quad \text { estimation interval, } \\
& \sigma_{d_{i}}^{2} \text { is the variance of the difference vector. }
\end{aligned}
$$

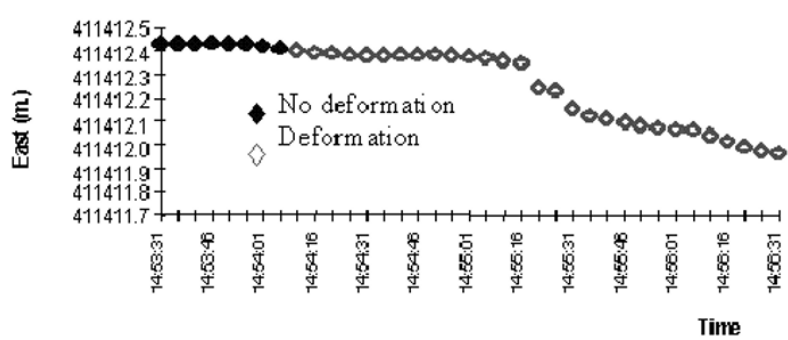

Fig. 1(a). Deformation detected with 5 second observations in E.

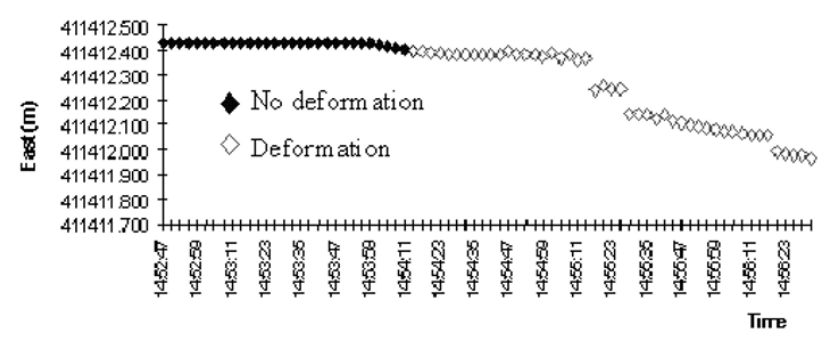

Fig. 1(b). Deformation detected with 3 second observations in E.

Actually, there is a mathematical correlation between the results of the $i$ th and 20th estimation intervals because of the recursive Kalman Filter algorithm. As in Eq. (11), the assumption is that there is no between-epoch correlation.

For deformation analysis, the zero and alternative hypotheses are:

$$
H_{0}: E\left\{d_{i}^{h}\right\}=0 \quad H_{a}: E\left\{d_{i}^{h}\right\} \neq 0 .
$$

If $T_{i}^{h} \geq F_{\left(1-a, t, f_{0}\right)}$, it is considered that the difference vector, $d_{i}^{h}$, is significant and that there is indeed a deformation in the height component. The analysis is also applied for the north and east components.

To test the software, the movements of a vehicle were continuously monitored with real-time kinematic GPS. The vehicle collected data in a stationary mode for sometime and then moved very slowly. Since the expected deformations are very small, the dynamic model of the filter is selected to be the motion with constant velocity. In the real-time application, the variance of the observations $\left(\sigma_{i}^{2}\right)$ and the variance of the non-deterministic variable in the dynamic model $\left(\sigma_{a}^{2}\right)$ are assumed to be $2 \mathrm{~cm}^{2}$ and $0.00001 \mathrm{~cm}^{2} / \mathrm{sec}^{4}$ respectively. The observation interval was one second. However, two estimation intervals are taken into consideration, which are 5 and 3 seconds. This means that each filtering step takes five or three observations into account. The software saves all the observations, filter and analysis results in a data base. So, in critical cases, the data set can be post-processed. The deformation analysis and the Kalman filtering are performed in three dimensions: north, east and height. The results are plotted in Figs. 1-3. The solid squares in the figures illustrate no deformations and the opaque squares shows the deformation period according to the 20 th estimation interval.

The obtained magnitudes for the deformation detection are \pm 3.5 and $\pm 3.0 \mathrm{~cm}$ for 3 and 5 second intervals respectively. Although the magnitudes are slightly different, the deforma- 


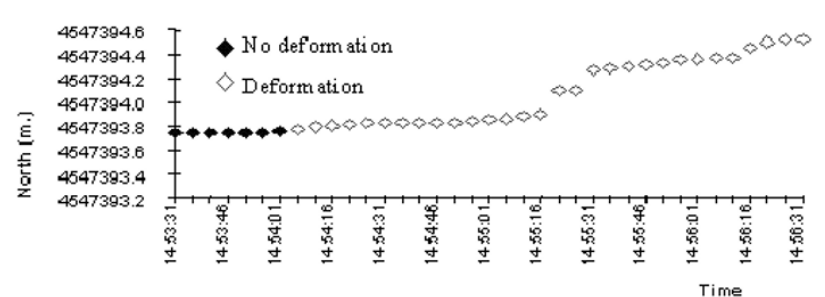

Fig. 2(a). Deformation detected with 5 second observations in N.

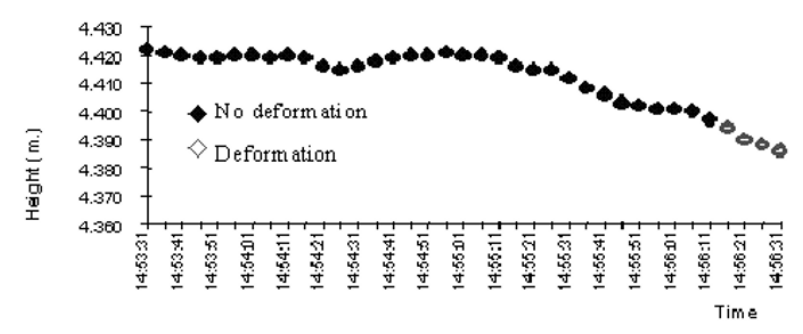

Fig. 3(a). Deformation detected with 5 second observations in $\mathrm{H}$.

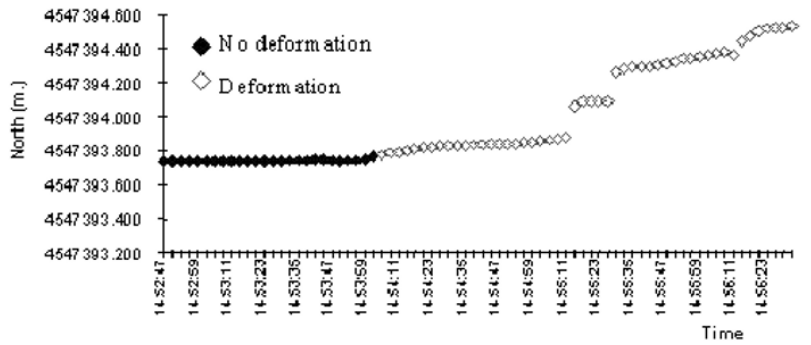

Fig. 2(b). Deformation detected with 3 second observations in N.

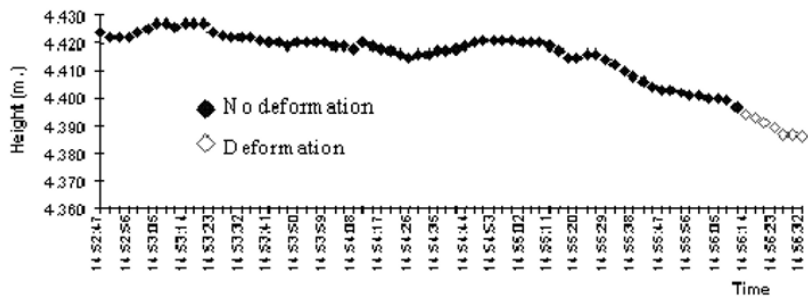

Fig. 3(b). Deformation detected with 3 second observations in $\mathrm{H}$.

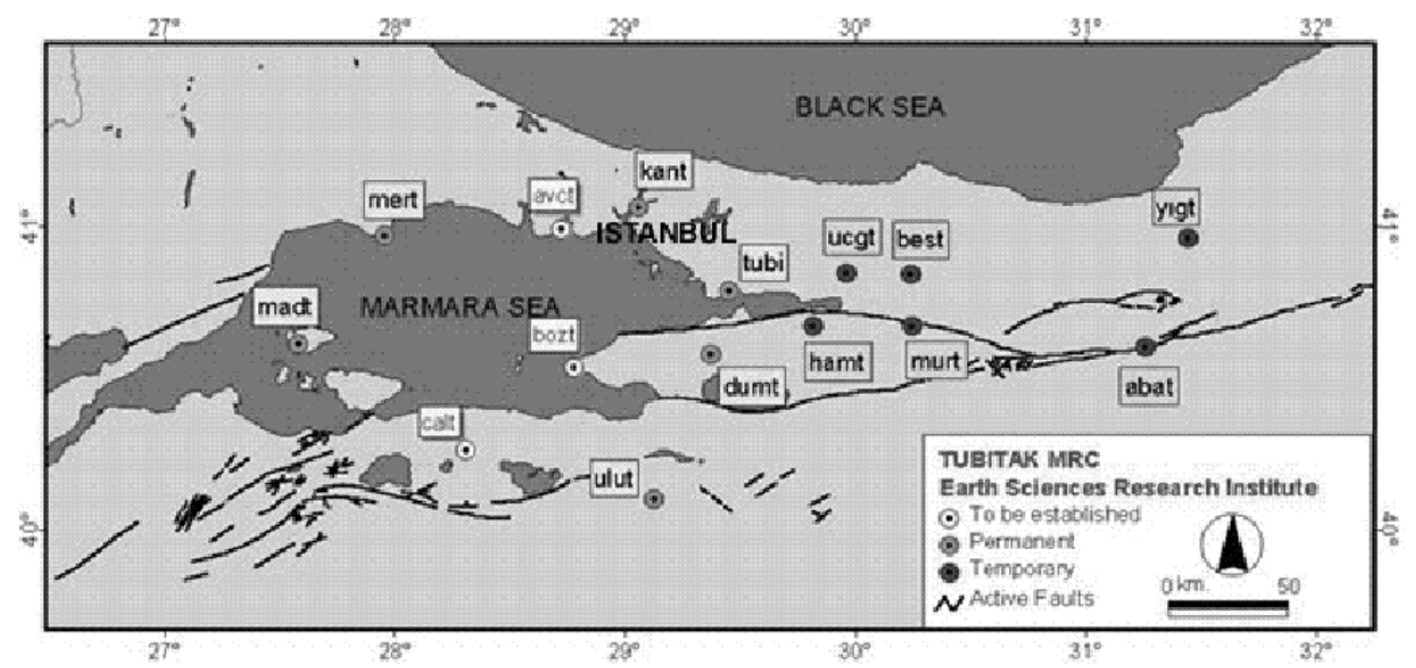

Fig. 4. Continuous GPS network in the Marmara Region in Turkey (TUBITAK-MAM).

tions were detected in the same epoch. It is obvious that the accuracy strongly depends on the number of observations taken into account in the analysis. On the other hand, the apriori values for the variances of the dynamic models are also important for the accuracy of deformation detection. For example, when the variance of the non-deterministic variable in the dynamic model $\left(\sigma_{a}^{2}\right)$ changed from 0.00001 to 0.000001 $\mathrm{cm}^{2} / \mathrm{sec}^{4}$, a movement of $\pm 2.3 \mathrm{~cm}$ was detected as deformation. Another criterion that affects the level of deformation detection is the number of observation used before the deformation analysis is permitted to start (in this study this number was 20).

Due to the page limitation in this article, the authors are not able to include the deformation analyses results. The full analysis results are found in Ince (1999), but unfortunately in the Turkish language.

\section{Continuous GPS Network in the Marmara Re- gion in Turkey}

Twenty million people, approximately one-third of the entire population of Turkey, live in and around Istanbul, and $40 \%$ of the Turkish industry is located in this region. Historical earthquake records of the area indicate that the region needs to be monitored closely using the latest technologies. For this purpose, a continuous GPS network has been established in and around Istanbul. The project, which was funded by the World Bank in 1997, is a collaborative effort of the Scientific and Technical Research Council of Turkey (TUBITAK), the Istanbul Technical University (ITU), the Bosphorus University and the General Command of Mapping. Each group in the project has specific responsibilities. At ITU, the authors are developing a real-time continuous deformation analysis software package, called RT-MODS2. The TUBITAK is responsible for the installation of the stations and for collecting GPS data in real-time. Fifteen GPS 
sites are proposed, located along the active faults of the region (Fig. 4). Currently, 12 sites are operational, but they do not permit analysis of observations in real-time.

\section{Concluding Remarks}

The main objective of this study was the development of a system for the investigation of catastrophes within the Marmara Region in Turkey. To satisfy this objective a software package (called RT-MODS2), which performs single station deformation analysis, has been developed based on the Kalman Filter technique. In order to test the software, very small movements of a vehicle have been successfully monitored. The software still needs be improved in order to perform a true network deformation analysis. For realtime applications such as this, it should be noted that the transmission of the data from all the GPS monitoring sites at pre-defined time intervals is required, in order to perform a real-time network deformation analysis.

\section{References}

Barka, A., The August 17 and November 121999 Earthquakes in the Eastern Marmara Sea Region, International Symposium on the Kocaeli Earthquake, Istanbul, December 2-5, 1999.

Bennet, R. A., B. P. Wernicke, and J. L. Davis, Continuous GPS measurements of contemporary deformation across the northern basin and range province, Geophys. Res. Lett., 25(4), 1998.

Bock, Y. and M. Bevis, Regional Permanent GPS Arrays. Trends and Prospects, International Symposium on GPS, Tsukuba, Japan, October 18-22, 1999.

Cross, P. A., Advanced Least Squares Applied to Position-Fixing, North East London Working paper No. 6 Polytechnic, Department of Land Surveying, 1990.
Grewal, M. S. and A. P. Andrews, Kalman Filtering Theory and Practice, 380 pp., Prentice Hall, Englewood Cliffs, New Jersey, 1993.

Hofmann-Wellenhof, B., H. Lichtenegger, and J. Collins, GPS Theory and Practice, 370 pp., 4th edition, Springer Verlag, Wien New York, 1997.

Ince, C. D., Real-time monitoring of Dynamic Systems with GPS and Kalman Filter, Ph.D. thesis, Istanbul Technical University, 154 pp., 1999 (in Turkish).

Jin, X. X., Theory of Carrier Adjusted DGPS Positioning Approach and Some Experimental Results, Delft University Press, Netherlands, 164 pp., 1996.

Kalman, R. E., A new approach to linear filtering and prediction problems, Journal of Basic Engineering, 82D, 35-45, 1960.

Kalman, R. E. and R. S. Bucy, New results in linear filtering and prediction theory, Journal of Basic Engineering, 83D, 95-108, 1961.

Langley, R. B., NMEA 0183: AGPS Receiver Interface Standard, GPS World, 54-57, July 1995.

Leick, A., GPS Satellite Surveying, John Wiley and Sons, Inc., 584 pp., 1995.

Maybeck, P. S., Stochastic Models, Estimation and Control Volume I, 423 pp., Academic Press, Inc., New York, 1979.

Salzmann, M., MDB: A Design Tool for Integrated Navigation Systems, Kinematic Systems in Geodesy, Surveying and Remote Sensing Symposium No. 107, Banff, Alberta, Canada, September 10-13, pp. 218-227, 1990

Teunissen, P. J. G., Some Aspects of Real-Time Validation Techniques for Use in Integrated Systems, Kinematic Systems in Geodesy, Surveying and Remote Sensing Symposium No. 107, Banff, Alberta, Canada, September 10-13, pp. 191-200, 1990.

Teunissen, P. J. G. and M. A. Salzmann, Performance Analysis of Kalman Filters, Reports 88.2, Delft University of Technology, The Faculty of Geodesy, Mathematical and Physical Geodesy, Netherlands, 1988.

Tsai, C. and L. Kurz, An adaptive robustizing approach to Kalman Filtering, Automatica, 19, 279-288, 1983.

C. D. Ince and M. Sahin (e-mail: sahin@itu.edu.tr) 